\title{
SOME CHARACTERIZATIONS OF INNER-PRODUCT SPACES
}

\author{
BY \\ MAHLON M. DAY
}

1. Introduction. The theorems of this paper give a number of conditions under which the norm in a real-linear or complex-linear normed space can be defined from an inner product. It should be emphasized that these are not conditions that the space have the same topological structure as an innerproduct space but are conditions that the existing norm $\|b\|$ in the space can be defined by the equation $\|b\|^{2}=(b, b)$ from an inner product satisfying the usual axioms.

The prototype of all these results is the von Neumann-Jordan condition $[11]\left(^{1}\right)$ that a linear metric space $B$ is an inner-product space if and only if $\left\|b+b^{\prime}\right\|^{2}+\left\|b-b^{\prime}\right\|^{2}=2\left(\|b\|^{2}+\left\|b^{\prime}\right\|^{2}\right)$ for every pair of points $b$ and $b^{\prime}$ in $B$. This implies that a space is an inner-product space if and only if every twodimensional subspace is an inner-product space; because of this fact most of the computations of this paper can be performed in two-dimensional spaces.

In a real two-dimensional space it is well known that the space is an innerproduct space if and only if the set of points of norm one is an ellipse; to prove sufficiency of our conditions in the real two-dimensional case it turns out to be convenient to have means of associating a specific ellipse with any given unit sphere and then to prove that, under the given conditions, the unit sphere is that particular ellipse. Two ways are used to select such an ellipse for several of these proofs.

(A) By Theorem 1 of the preceding paper [5], if $C$ is the set of points of norm one, there is a parallelogram $P$ circumscribed about $C$ so that the midpoint of each side of $P$ is on $C$. The largest ellipse $E$ inscribed in $P$ also meets the midpoints of the sides of $P$. Under the hypotheses of Theorems 2.1 and 3.1 it can be shown that $C$ is $E$.

(B) Loewner has shown (in some unpublished work) that there exists a unique ellipse $E^{\prime}$ of minimal area circumscribed about $C$, and that this ellipse touches $C$ in at least four points. Under the conditions of Theorems 2.1 and 4.1 , it is possible to show that $C$ is $E^{\prime}$.

It turns out that the real-linear cases of the following theorems can be proved more easily than the complex-linear cases; this is due to the availability of the geometrical devices (A) and (B) in the real two-dimensional case. The proofs for the real-linear cases are given in $\$ \$ 2$ through 6 .

In $\$ 7$ the complex cases of these theorems are derived from the real cases

Presented to the Society, April 29, 1944; received by the editors September 27, 1946.

(1) Numbers in brackets refer to the bibliography at the end of the paper. 
and from certain elementary but interesting relationships between a complexlinear space $B$ and the real-linear space $A$ associated with it by forgetting to use the non-real scalar multiples. For example, Theorem 7.1 says that there is a natural, one-to-one, real-linear, norm-preserving mapping between all of $A^{*}$, the space of real-valued, real-linear functions on $A$, and all of $B^{*}$, the space of complex-valued, complex-linear functions on $B$. Theorem 7.2 says that the norm in a complex-linear normed space $B$ arises from a complex inner product if and only if it arises from a real inner product. These results easily give the complex cases of the theorems 1 to 7 from the real cases which are to be proved in $\S \S 2$ to 6 .

The first of the criteria to be given is a formal weakening of the condition given by Jordan and von Neumann in complex-linear metric spaces. If the space is assumed to be normed, it is obvious that one of the points $b$ and $b^{\prime}$ used in the test condition can be chosen to be of norm one. The first theorem of this paper states that the additional restriction that both points are of norm one can be imposed (Theorem 2.1).

Further theorems give characterizations in terms of: a condition using points both of $B$ and its conjugate space (Theorem 3.1), uniform convexity (Theorem 4.1), various types of orthogonality (Theorems 5.1 and 5.2) (completing certain results of James [9]), and normality (extending a result of G. Birkhoff [2]).

2. A weakening of the von Neumann-Jordan condition. We begin with the definition of a real inner product space; we shall be concerned in the next five sections only with the real linear case; the complex case and its relation to the real is given in $\$ 7$. A (real) inner product in a real-linear $\left.{ }^{2}\right)$ space $B$ is a real-valued function of two variables $\left(b, b^{\prime}\right)$ in $B$ satisfying the conditions:

R1. $(b, b)>0$ if $b \neq 0$;

R2. $\left(b, b^{\prime}\right)=\left(b^{\prime}, b\right)$;

R3. $\left(m_{1} b_{1}+m_{2} b_{2}, b_{3}\right)=m_{1}\left(b_{1}, b_{3}\right)+m_{2}\left(b_{2}, b_{3}\right)$ for all elements $b_{i}$ of $B$ and real numbers $m_{i}$.

A real-linear normed space $B$ is a real inner-product space if there exists a real inner product such that $\|b\|^{2}=(b, b)$ for every $b$ in $B$.

THEOREM 2.1. A normed space $B$ is an inner-product space if and only if it satisfies the condition

$$
\left\|b_{1}+b_{2}\right\|^{2}+\left\|b_{1}-b_{2}\right\|^{2}=4 \quad \text { if }\left\|b_{1}\right\|=\left\|b_{2}\right\|=1 .
$$

It is well known that a two-dimensional normed space is an inner-product space if and only if the unit sphere in $B$ is an ellipse; from this we see that a special case of 2.1 is the following theorem.

(2) A linear space is an Abelian group $B$ (written additively) in which multiplication by scalars is defined to satisfy certain associative and distributive laws (see Banach [1]). If the scalars are real (complex) numbers, $B$ will be called a real-linear (complex-linear) space. 
THEOREM 2.2. If $B$ is a two-dimensional real-linear space and $C$ is the set of points of norm one, $C$ is an ellipse if and only if $\left(^{*}\right)$ holds.

Two methods of proof are available for this theorem. One begins with the circumscribed ellipse of smallest area and proves that it is $C$. A second proof using the method (A) of the introduction was discovered earlier but takes several times as much space; it will be outlined at the end of this section. The first proof uses some lemmas due to Loewner.

Lemma 2.3 (LOEWNeR). If $C$ is a symmetric closed convex curve, there exists a unique ellipse of minimal area circumscribed about $C$.

Let $E_{1}$ and $E_{2}$ be ellipses of minimal area circumscribed about $C$, make an affine transformation reducing both ellipses to principal axes so that equations for them may be written in the form $x^{2}+y^{2}=1, a x^{2}+b y^{2}=1$. Since these are of equal area, $\pi, a b=1$. The condition that they contain $C$ shows that $x^{2}+y^{2} \leqq 1$ and $a x^{2}+b y^{2} \leqq 1$ for $(x, y)$ on $C$. Hence $(a+1) x^{2} / 2+(b+1) y^{2} / 2 \leqq 1$ also for points of $C$; that is, the ellipse $(a+1) x^{2} / 2+(b+1) y^{2} / 2=1$ contains $C$. The area of this last ellipse is $2 \pi /((a+1)(b+1))^{1 / 2}$. This is less than $\pi$ unless $a=b=1$ so the minimal ellipse is unique.

LEMMa 2.4 (LOEWNeR). The minimal ellipse circumscribed about a symmetric closed convex $C$ touches $C$ in at least four points.

If the minimal ellipse $E$ is written in the form $x^{2}+y^{2}=1$, where $(1,0)$ is a point of contact of $C$ and $E$, then the ellipses $E_{\epsilon}$ with equations $x^{2} /(1+\epsilon)$ $+(1+\epsilon) y^{2}=1$ also have the minimal area so do not contain $C$. For each $\epsilon>0$ let $p_{\epsilon}$ be a point of $C$ not in $E_{\epsilon}$; then there is a sequence $\epsilon_{n} \rightarrow 0$ such that $p_{\epsilon_{n}}$ converges to some point $p$. Since all $p_{\epsilon}$ are on $C, p$ is on $C$; moreover $p_{\epsilon}$ is within $\epsilon$ of $E$, so $p$ is also on $E$. Now the intersections of $E$ and $E_{\epsilon}$ have $y$ coordinates $\pm 1 /(2+\epsilon)^{1 / 2}$; hence the absolute value of the $y$ coordinate of $p$ is at least $1 / 2^{1 / 2}$. Therefore $p$ is not either point $( \pm 1,0)$ and is on both $C$ and $E$. By symmetry there are at least four points of contact of $C$ and $E$.

To apply Lemma 2.4 to the proof of Theorem 2.2 , let $C$ be the set of points $b$ for which $\|b\|=1$ and let $E$ be the ellipse of minimal area circumscribed about $C$. Also define a new norm $|b|$ in $B$ so that $E$ is the set of all those $b$ for which $|b|=1$. Since $|b|$ can be defined from an inner product, the relation $\left(^{*}\right)$ holds for $|b|$. Since $C$ is inside $E,\|b\| \geqq|b|$ for every $b$. Now let $x$ and $y \neq \pm x$ be two points of contact of $C$ and $E$. Then $4=|x+y|^{2}+|x-y|^{2}$ $\leqq\|x+y\|^{2}+\|x-y\|^{2}=4$; hence $\|x+y\|=|x+y|$ and $\|x-y\|=|x-y|$. That is to say, if $C$ and $E$ meet at two points, then they meet at two more points roughly half way between the original points.

Repeating the process by induction shows that $C$ and $E$ agree on a dense set of points; hence $C$ is $E$. 2.2 now implies 2.1 by the von Neumann-Jordan condition.

The second proof of Theorem 2.2 is too long to give here in full but will be 
sketched instead. By Theorem 1 of my preceding paper [5] there exists a parallelogram $P$ circumscribed about $C$ so that the midpoint of each side of $P$ is on $C$. Make an affine transformation of $B$ that carries $P$ into a square and draw the inscribed circle $E$ of $P$. We wish to show that when $\left(^{*}\right)$ holds, $C$ is $E$. Represent $C$ by the polar coordinate equation $r=f(\theta)$ where the polar coordinate system is so chosen that $\theta=0$ is a line from the origin to the midpoint of one side of $P$. The major part of the proof is the proof of the following lemma.

LEMma 2.5. If $\left(^{*}\right)$ holds, if $0 \leqq \theta_{0}<\theta_{1} \leqq \pi / 2$, and if $f\left( \pm \theta_{0}\right)=1=f\left( \pm \theta_{1}\right)$, then $f\left(\left(\theta_{0}+\theta_{1}\right) / 2\right)=f\left(-\left(\theta_{0}+\theta_{1}\right) / 2\right)=f\left(\pi / 2-\left(\theta_{0}+\theta_{1}\right) / 2\right)=f\left(-\pi / 2+\left(\theta_{0}+\theta_{1}\right) / 2\right)=1$.

To prove this lemma let $\phi=\left(1, \theta_{0}\right), \psi=\left(1, \theta_{1}\right), \Phi=\left(1,-\theta_{0}\right)$, and $\Psi$ $=\left(1,-\theta_{1}\right)$. Define $k_{1}=|\phi+\psi| / 2=|\Phi+\Psi| / 2=\cos \left(\theta_{1}-\theta_{0}\right) / 2$ and $k_{2}$ $=|\phi-\psi| / 2=|\Phi-\Psi| / 2=\cos \left[\pi / 2-\left(\theta_{1}-\theta_{0}\right) / 2\right]$. Then let $r_{1}=f\left(\left(\theta_{0}+\theta_{1}\right) / 2\right)$, $r_{2}=f\left(\pi / 2-\left(\theta_{0}+\theta_{1}\right) / 2\right), \quad R_{1}=f\left(-\left(\theta_{0}+\theta_{1}\right) / 2\right)$, and $R_{2}=f\left(-\pi / 2+\left(\theta_{0}+\theta_{1}\right) / 2\right)$. Then $\|\phi+\psi\| / 2=k_{1} / r_{1},\|\phi-\psi\| / 2=k_{2} / R_{2},\|\Phi+\Psi\| / 2=k_{1} / R_{1}$, and $\|\Phi-\Psi\| / 2$ $=k_{2} / r_{2}$.

Dividing $\left(^{*}\right)$ by 4 and substituting from the equations just above gives two conditions on these variables:

$$
\begin{aligned}
& k_{1}^{2} / r_{1}^{2}+k_{2}^{2} / R_{2}^{2}=1, \\
& k_{1}^{2} / R_{1}^{2}+k_{2}^{2} / r_{2}^{2}=1 .
\end{aligned}
$$

Let $\theta^{\prime}=\left(\theta_{0}+\theta_{1}\right) / 2, \phi^{\prime}=\left(r_{1}, \theta^{\prime}\right)$, and $\Phi^{\prime}=\left(R_{1},-\theta^{\prime}\right)$. The horizontal projection of $\phi^{\prime}+\Phi^{\prime}$ is $r_{1} \cos \theta^{\prime}+R_{1} \cos \left(-\theta^{\prime}\right)=\left(r_{1}+R_{1}\right) \cos \theta^{\prime}$. Since $C$ is inside $P$, $\left\|\phi^{\prime}+\Phi^{\prime}\right\| \geqq\left(r_{1}+R_{1}\right) \cos \theta^{\prime}$. A similar argument with the vertical coordinates shows that $\left\|\phi^{\prime}-\Phi^{\prime}\right\| \geqq\left(r_{1}+R_{1}\right) \sin \theta^{\prime}$. Similar arguments with $\psi^{\prime}=\left(r_{2}, \pi / 2-\theta^{\prime}\right)$ and $\Psi^{\prime}=\left(R_{2}, \theta^{\prime}-\pi / 2\right)$ give $\left\|\psi^{\prime}+\Psi^{\prime}\right\| \geqq\left(r_{2}+R_{2}\right) \cos \left(\pi / 2-\theta^{\prime}\right)$ and $\left\|\psi^{\prime}-\Psi^{\prime}\right\|$ $\geqq\left(r_{2}+R_{2}\right) \sin \left(\pi / 2-\theta^{\prime}\right)$. Substituting in $\left(^{*}\right)$ gives $\left(r_{1}+R_{1}\right)^{2} \leqq 4$ and $\left(r_{2}+R_{2}\right)^{2}$ $\leqq 4$ or

$$
\begin{aligned}
& r_{1}+R_{1} \leqq 2 \\
& r_{2}+R_{2} \leqq 2 .
\end{aligned}
$$

It can be shown by straightforward computation that the only solution of these four conditions is $r_{1}=r_{2}=R_{1}=R_{2}=1$; to prove this it suffices by the symmetry of the equations to show that no solution exists with $r_{1}>1$. This shows that the points $\phi^{\prime}, \psi^{\prime}, \Phi^{\prime}$, and $\Psi^{\prime}$ are on $E$ as well as on $C$.

This lemma says that if $C$ and $E$ touch in two symmetric pairs of points, then they touch at some intermediate symmetric pairs of points. Starting with $\theta_{0}=0, \theta_{1}=\pi / 2$, and applying this lemma repeatedly shows that $C$ and $E$ coincide on a dense set; hence $C$ is $E$.

3. A characterization by an equation involving points of both $B$ and its conjugate space. In the paper [6] I proved the relation that if $\left\|b_{i}\right\|=\left\|\beta_{i}\right\|$ 
$=\beta_{i}\left(b_{i}\right)=1$ for $i=1,2$, then $8 \geqq\left\|\beta_{1}+\beta_{2}\right\| \cdot\left\|b_{1}+b_{2}\right\|+\left\|\beta_{1}-\beta_{2}\right\| \cdot\left\|b_{1}-b_{2}\right\| \geqq 4$. The proof is trivial for

$$
\begin{aligned}
8=2 \cdot 2+2 \cdot 2 & \geqq\left\|\beta_{1}+\beta_{2}\right\| \cdot\left\|b_{1}+b_{2}\right\|+\left\|\beta_{1}-\beta_{2}\right\| \cdot\left\|b_{1}-b_{2}\right\| \\
& \geqq\left(\beta_{1}+\beta_{2}\right)\left(b_{1}+b_{2}\right)+\left(\beta_{1}-\beta_{2}\right)\left(b_{1}-b_{2}\right) \\
& =2\left(\beta_{1}\left(b_{1}\right)+\beta_{2}\left(b_{2}\right)\right)=4 .
\end{aligned}
$$

Since the last inequality can not always be replaced by equality, a natural problem is the characterization of those $B$ where equality holds for all such $b_{i}$ and $\beta_{i}$. This problem is solved by the following theorem.

THEOREM 3.1. Each of the following conditions is necessary and sufficient that a normed space $B$ be an inner-product space:

$$
\begin{aligned}
& \left\|\beta_{1}+\beta_{2}\right\| \cdot\left\|b_{1}+b_{2}\right\|+\left\|\beta_{1}-\beta_{2}\right\| \cdot\left\|b_{1}-b_{2}\right\|=4 \\
& \quad \text { whenever }\left\|b_{i}\right\|=\left\|\beta_{i}\right\|=\beta_{i}\left(b_{i}\right)=1 \text { for } i=1,2 . \\
& \left(\beta_{1}+\beta_{2}\right)\left(b_{1}+b_{2}\right)=\left\|\beta_{1}+\beta_{2}\right\| \cdot\left\|b_{1}+b_{2}\right\| \\
& \quad \text { whenever }\left\|b_{i}\right\|=\left\|\beta_{i}\right\|=\beta_{i}\left(b_{i}\right)=1 \text { for } i=1,2 .
\end{aligned}
$$

If $\left(^{* *}\right)$ holds, we see from the proof above that

$$
\begin{array}{r}
\left\|\beta_{1}+\beta_{2}\right\| \cdot\left\|b_{1}+b_{2}\right\|+\left\|\beta_{1}-\beta_{2}\right\| \cdot\left\|b_{1}-b_{2}\right\| \\
=\left(\beta_{1}+\beta_{2}\right)\left(b_{1}+b_{2}\right)+\left(\beta_{1}-\beta_{2}\right)\left(b_{1}-b_{2}\right) .
\end{array}
$$

Since each term on the left is as large as the corresponding term on the right, we see that $\left(^{* *}\right)$ implies $\left({ }^{* * *}\right)$. Conversely $\left({ }^{* * *}\right)$, applied to $\beta_{1},-\beta_{2}, b_{1}$, and $-b_{2}$ as well, gives $\left({ }^{* *}\right)$ by addition so these two conditions are equivalent.

Geometrically the condition that $\beta(b)=\|\beta\| \cdot\|b\|$ means that the hyperplane $\left(^{3}\right)\left\{b^{\prime} \mid \beta\left(b^{\prime}\right)=\|\beta\| \cdot\|b\|\right\}$ has no points nearer to the origin than $b$; that is, the hyperplane $\left\{b^{\prime} \mid \beta\left(z^{\prime}\right) /\|\beta\|=1\right\}$ is a hyperplane of support to the unit sphere at $\left.b /\|b\| .{ }^{* * *}\right)$ asserts that a hyperplane of support at $\left(b_{1}+b_{2}\right) /\left\|b_{1}+b_{2}\right\|$ can be found in terms of the hyperplanes of support at $b_{1}$ and $b_{2}$ on the unit sphere.

To begin the proof of Theorem 3.1 recall that, in an inner-product space $B$, for some $\beta^{\prime}$ s in $B^{*}$ there exist $b_{\beta}$ in $B$ for which $\left(b^{\prime}, b_{\beta}\right)=\beta\left(b^{\prime}\right)$ for all $b^{\prime}$ in $B$ (if $B$ is complete $b_{\beta}$ exists for every $\beta$ ). If $b_{\beta_{1}}$ and $b_{\beta_{2}}$ exist, $b_{\beta_{1}+\beta_{2}}$ exists equal to $b_{\beta_{1}}+b_{\beta_{2}}$. Also, whenever $\|\beta\|=\|b\|=\beta(b)=1$, it follows that $b_{\beta}=b$. From this and $\left(^{*}\right)$ we can show that if $B$ is an inner-product space, then $\left({ }^{* *}\right)$ holds. If $\left\|b_{i}\right\|=\left\|\beta_{i}\right\|=\beta_{i}\left(b_{i}\right)=1$, then $\left\|\beta_{1}+\beta_{2}\right\|=\left\|b_{\beta_{1}+\beta_{2}}\right\|=\left\|b_{\beta_{1}}+b_{\beta_{2}}\right\|=\left\|b_{1}+b_{2}\right\|$. A similar relation holds for differences; hence

$$
\begin{aligned}
\left\|\beta_{1}+\beta_{2}\right\| \cdot\left\|b_{1}+b_{2}\right\|+\left\|\beta_{1}-\beta_{2}\right\| \cdot\left\|b_{1}-b_{2}\right\| \\
=\left\|b_{1}+b_{2}\right\|^{2}+\left\|b_{1}-b_{2}\right\|^{2}=4 \text { by }
\end{aligned}
$$

(') The symbol $\{p \mid Q\}$ means the set of all $p$ having the property $Q$. 
To prove $\left({ }^{* *}\right)$ or the equivalent condition $\left({ }^{* * *}\right)$ sufficient we begin, as usual, with the real, two-dimensional case.

THEOREM 3.2. If $B$ is a two-dimensional normed space and $C$ is the set of points of norm one, then $C$ is an ellipse if and only if $\left(^{* *}\right)$ holds; that is, if and only if $(* * *)$ holds.

As in the proof sketched in $\$ 2$ we begin by applying Theorem 1 of the preceding paper to $C$, turning the resulting parallelogram into a square $P$, and defining $E$ to be the circle inscribed in $P$. As before, define $|b|$ so that $E=\{b|| b \mid=1\}$ and set up polar coordinates $(r, \theta)$ so that $r=|b|$ and $(1,0)$ is the midpoint of a side of $P$. Let $r(b)$ and $\theta(b)$ be the polar coordinates of $b$.

If $B_{1}$ is the space with the same elements as $B$ but with $|b|$ for norm, $B_{1}$ is a complete inner-product space; hence if $\beta$ is in $B_{1}^{*}$, there exists $b_{\beta}$ in $B_{1}$ such that $\left(b, b_{\beta}\right)=\beta(b)$ for all $b$ in $B_{1}$. (Here $\left(b, b^{\prime}\right)$ is the inner product associated with $|b|$.) Let the polar coordinates of $b_{\beta}$ be $(r(\beta), \theta(\beta))$; then $r(\beta)$ $=|\beta|=\left|b_{\beta}\right|$ and $\theta(\beta)=\theta\left(b_{\beta}\right)$.

It will be helpful in computing values of $r(\beta)$ and $\theta(\beta)$ if we give a second way of locating $b_{\beta}$. It is an elementary extension of the discussion of $b_{\beta}$ to show that if $b_{\beta}^{\prime}$ is that (unique) point of $\{b \mid \beta(b)=1\}$ of minimum $|b|$, then $r(\beta)=\left|b_{\beta}\right|=1 /\left|b_{\beta}^{\prime}\right|$ and $\theta(\beta)=\theta\left(b_{\beta}^{\prime}\right)$.

From this we can show that if $\|\beta\|=\|b\|=\beta(b)=1$ and $-\pi / 2 \leqq \theta(b) \leqq \pi / 2$, then $\theta(\beta)$ is a weakly nondecreasing function of $\theta(b)$; that is, if $\theta\left(b_{1}\right)>\theta\left(b_{2}\right)$, then $\theta\left(\beta_{1}\right) \geqq \theta\left(\beta_{2}\right)$. This is an immediate consequence of the convexity of $C$; if $C$ has no corners, $\theta(\beta)$ is even a single-valued function of $\theta(b)$ and $\theta\left(\beta_{1}\right)$ $\geqq \theta\left(\beta_{2}\right)$ if $\theta\left(b_{1}\right) \geqq \theta\left(b_{2}\right)$; if, moreover, $C$ has no flat sides $\theta(\beta)$ increases strictly with $\theta(b)$.

With these preliminaries we are now prepared to start a proof that $C$ is $E$. This proof requires a number of steps, the first of which is an induction argument showing that $C$ is symmetric about the line $\theta=0$. Using this fact and another induction argument, we show that if $\theta=m \pi / 2^{n}, m$ and $n$ integers, then the point $(1, \theta)$ is on $C$ as well as on $E$. Since $C$ is convex, this will show that $C$ and $E$ coincide.

The lemma to be used in the first induction says, roughly, that if the curve $C$ with its tangent directions is symmetric about $\theta=0$ at two pairs of points, then the curve and its tangent directions are symmetric about $\theta=0$ at a pair of points between the first pairs.

Lemma 3.3. Suppose that $\left({ }^{* * *}\right)$ holds and that, for $i=1$ and 2 , points $s_{i}$ and $s_{i}^{\prime}$ in $B$ and points $\sigma_{i}$ and $\sigma_{i}^{\prime}$ in $B^{*}$ can be chosen so that (a) $\left\|s_{i}\right\|=\left\|\sigma_{i}\right\|=\sigma_{i}\left(s_{i}\right)$ $=1=\left\|s_{i}^{\prime}\right\|=\left\|\sigma_{i}^{\prime}\right\|=\sigma_{i}^{\prime}\left(s_{i}^{\prime}\right)$; (b) $\theta\left(s_{i}^{\prime}\right)=-\theta\left(s_{i}\right)$ and $\theta\left(\sigma_{i}^{\prime}\right)=-\theta\left(\sigma_{i}\right), r\left(s_{i}^{\prime}\right)=r\left(s_{i}\right)$ and $r\left(\sigma_{i}^{\prime}\right)=r\left(\sigma_{i}\right)$, and (c) $\pi / 2 \geqq \theta\left(s_{2}\right)>\theta\left(s_{1}\right) \geqq 0, \pi / 2 \geqq \theta\left(\sigma_{2}\right)>\theta\left(\sigma_{1}\right) \geqq 0$; define $s=\left(s_{1}+s_{2}\right) /\left\|s_{1}+s_{2}\right\|, \quad s^{\prime}=\left(s_{1}^{\prime}+s_{2}^{\prime}\right) /\left\|s_{1}^{\prime}+s_{2}^{\prime}\right\|, \quad \sigma=\left(\sigma_{1}+\sigma_{2}\right) /\left\|\sigma_{1}+\sigma_{2}\right\|, \quad$ and $\sigma^{\prime}$ $=\left(\sigma_{1}^{\prime}+\sigma_{2}^{\prime}\right) /\left\|\sigma_{1}^{\prime}+\sigma_{2}^{\prime}\right\|$. Then $, s^{\prime} s^{\prime}, \sigma$, and $\sigma^{\prime}$ are related as are $s_{i}, s_{i}^{\prime}, \sigma_{i}$, and $\sigma_{i}^{\prime}$ 
for $i=1$ or 2 ; that is, $\left(\mathrm{a}^{\prime}\right)\|s\|=\|\sigma\|=\sigma(s)=1=\left\|s^{\prime}\right\|=\left\|\sigma^{\prime}\right\|=\sigma^{\prime}\left(s^{\prime}\right)$, and $\left(\mathrm{b}^{\prime}\right) r\left(s^{\prime}\right)=r(s), r\left(\sigma^{\prime}\right)=r(\sigma), \theta\left(s^{\prime}\right)=-\theta(s)$, and $\theta\left(\sigma^{\prime}\right)=-\theta(\sigma)$.

$s, s^{\prime}, \sigma$, and $\sigma^{\prime}$ are constructed to have the proper norms.

$$
\sigma(s)=\left(\sigma_{1}+\sigma_{2}\right)\left(s_{1}+s_{2}\right) /\left(\left\|\sigma_{1}+\sigma_{2}\right\| \cdot\left\|s_{1}+s_{2}\right\|\right)=1
$$

by $\left({ }^{* * *}\right)$; similarly $\sigma^{\prime}\left(s^{\prime}\right)=1$ so $\left(a^{\prime}\right)$ holds. By the symmetry of the original conditions $\theta(s)=\theta\left(s_{1}+s_{2}\right)=-\theta\left(s_{1}^{\prime}+s_{2}^{\prime}\right)=-\theta\left(s^{\prime}\right)$; similarly $\theta\left(\sigma^{\prime}\right)=-\theta(\sigma)$.

To prove that $r\left(s^{\prime}\right)=r(s)$ recall that $b_{\beta}^{\prime}$ was defined to be the point of least absolute value on the line $\{b \mid \beta(b)=1\}$; also $\theta\left(b_{\beta}^{\prime}\right)=\theta(\beta)$. By the geometric meaning of $\left(\mathrm{a}^{\prime}\right)$ we see that $r\left(b_{\sigma}^{\prime}\right)=r(s) \cos [\theta(s)-\theta(\sigma)]$ and $r\left(b_{\sigma^{\prime}}^{\prime}\right)=r\left(s^{\prime}\right)$ $\cos \left[\theta\left(s^{\prime}\right)-\theta\left(\sigma^{\prime}\right)\right]$. Now suppose that $r(s)>r\left(s^{\prime}\right)$ then, since $\theta(s)-\theta(\sigma)=$ $-\left[\theta\left(s^{\prime}\right)-\theta\left(\sigma^{\prime}\right)\right]$, we see that $r\left(b_{\sigma}^{\prime}\right)>r\left(b_{\sigma^{\prime}}^{\prime}\right)$ or, taking reciprocals, $r\left(b_{\sigma}\right)<r\left(b_{\sigma^{\prime}}\right)$. Now let $b_{0}=(1,0)=b_{\beta_{0}}$; then $\theta\left(s+s^{\prime}\right)>0=\theta\left(b_{0}\right)$; also $\theta\left(\sigma+\sigma^{\prime}\right)=\theta\left(b_{\sigma}+b_{\sigma^{\prime}}\right)$ $<0=\theta\left(\beta_{0}\right)$. However, by $\left(^{* * *}\right)$ we have $\left(\sigma+\sigma^{\prime}\right)\left(s+s^{\prime}\right)=\left\|\sigma+\sigma^{\prime}\right\| \cdot\left\|s+s^{\prime}\right\|$, or the line $\left\{b \mid \sigma(b)+\sigma^{\prime}(b)=\left\|\sigma+\sigma^{\prime}\right\|\right\}$ is a line of support to $C$ at $\left(s+s^{\prime}\right) /\left\|s+s^{\prime}\right\|$. However, for $b$ and $\beta$ to be related in this way, $\theta(\beta)$ increases with $\theta(b)$ so $\theta\left(\sigma+\sigma^{\prime}\right) \geqq 0$ when $\theta\left(s+s^{\prime}\right)>0$. This contradiction shows that $r(s) \leqq r\left(s^{\prime}\right)$; a dual argument shows that $r(s) \geqq r\left(s^{\prime}\right)$ so equality holds here. Hence $r\left(b_{\sigma}^{\prime}\right)$ $=r\left(b_{\sigma^{\prime}}^{\prime}\right)$ so $r\left(\sigma^{\prime}\right)=r(\sigma)$. This completes the proof of $\left(\mathrm{b}^{\prime}\right)$.

We now apply this lemma to the proof of the following lemma.

LeMmA 3.4. If $\left(^{* * *}\right)$ holds, $C$ is symmetric about the line $\theta=0$.

In 3.3 first take $s_{1}=s_{1}^{\prime}=b_{\sigma_{1}}=b_{\sigma_{1}^{\prime}}=(1,0), s_{2}=b_{\sigma_{2}}=(1, \pi / 2)$, and $s_{2}^{\prime}=b_{\sigma_{2}}{ }^{\prime}$ $=(1,-\pi / 2)$; Lemma 3.3 then asserts that if $r$ is so chosen that $(r, \pi / 4)$ is on $C$, so is $(r,-\pi / 4)$ and also that the lines of inclination $\mp \pi / 4$, respectively, are lines of support at these points. Using the same choice of $s_{1}, s_{1}^{\prime}, \sigma_{1}$, and $\sigma_{1}^{\prime}$, and using these new points for $s_{2}$, and so on, we get two more points about half way between these which are also fully symmetric about $\theta=0$. Repeating by induction gives a dense set of values of $\theta, 0 \leqq \theta \leqq \pi / 2$, such that $(r, \theta)$ is on $C$ if and only if $(r,-\theta)$ is on $C$. By symmetry about the origin and continuity of $C$ it follows immediately that $C$ is symmetric about the line $\theta=0$.

From this we easily derive the following lemma.

Lemma 3.5. If (***) holds, if $P$ is a square circumscribed about $C$ so that the midpoint of each side of $P$ is on $C$, and if $\left(r_{0}, \theta_{0}\right)$ is the midpoint of one side of $P$, then $C$ is symmetric about the line $\theta=\theta_{0}$.

This follows from 3.4 since we used no other property of the direction $\theta=0$ than that described in the hypotheses of this lemma.

We saw in the proof of 3.4 that the lines of support of inclination $\pm \pi / 4$ form a square circumscribed about $C$ in the proper way; it follows from 3.5 that $C$ is symmetric about the lines $\theta=m \pi / 4, m=0,1,2$, and 3 .

One more lemma is needed. 
LEMMA 3.6. If (***) holds and if $\theta_{0}, 0<\theta_{0} \leqq \pi / 4$, is so selected that $C$ is symmetric about the line $\theta=2 \theta_{0}$ and so that $\left(1,2 \theta_{0}\right)$ is on $C$ as well as on $E$, then $\left(1, \theta_{0}\right)$ is on $C$ and $C$ is symmetric about the line $\theta=\theta_{0}$.

To prove symmetry about $\theta=\sigma_{0}$ define $a_{0}=(1,0)=b_{\alpha_{0}}$ and $a_{1}=\left(1,2 \theta_{0}\right)$ $=b_{\alpha_{1}}$; then, by symmetry about $\theta=0$ and $\theta=2 \theta_{0}$, we see that $\left\|a_{i}\right\|=\left\|\alpha_{i}\right\|$ $=\alpha_{i}\left(a_{i}\right)=1$. Let $b_{0}=\left(a_{0}+a_{1}\right) /\left\|a_{0}+a_{1}\right\|$ and $\beta_{0}=\left(\alpha_{0}+\alpha_{1}\right) /\left\|\alpha_{0}+\alpha_{1}\right\| ;$ then $\theta\left(b_{0}\right)=\theta_{0}$ since $r\left(a_{0}\right)=r\left(a_{1}\right) ; \theta\left(\beta_{0}\right)=\theta_{0}$; and by $\left({ }^{* * *}\right) \beta_{0}\left(b_{0}\right)=\left\|\beta_{0}\right\|=\left\|b_{0}\right\|=1$. If $b_{0}=\left(r_{0}, \theta_{0}\right)$, then $b_{\beta_{0}}=\left(1 / r_{0}, \theta_{0}\right)$. Let $d=\left(r_{0}, \theta_{0}+\pi / 2\right)$ and $b_{s}=\left(1 / r_{0}, \theta_{0}+\pi / 2\right)$. By symmetry of $C$ about $\theta=\pi / 4$ and $\theta=\pi / 2$, it follows that $\|d\|=\|\delta\|=\delta(d)=1$. Hence the lines of support of inclination $\theta_{0}$ and $\theta_{0}+\pi / 2$ form a square circumscribed about $C$ with midpoints at $\pm b_{0}$ and $\pm d$. By 3.5, $C$ is symmetrical about $\theta=\theta_{0}$.

Let $b_{1}=\left(r_{0},-\theta_{0}\right)$ and $b_{\beta_{1}}=\left(1 / r_{0},-\theta_{0}\right)$; by symmetry about $\theta=0, \beta_{1}\left(b_{1}\right)$ $=\left\|\beta_{1}\right\|=\left\|b_{1}\right\|=1$ also.

Let $c_{0}=\left(a_{0}+b_{0}\right) /\left\|a_{0}+b_{0}\right\|, c_{1}=\left(a_{0}+b_{1}\right) /\left\|a_{0}+b_{1}\right\|$ and $c_{2}=\left(a_{1}+b_{0}\right) /\left\|a_{1}+b_{0}\right\|$. By symmetry of $C$ about $\theta=0$ and $\theta=\theta_{0}$, we have $r\left(c_{0}\right)=r\left(c_{1}\right)=r\left(c_{2}\right), \theta\left(c_{1}\right)$ $=-\theta\left(c_{0}\right), \theta\left(c_{2}\right)=2 \theta_{0}-\theta\left(c_{0}\right)$. Defining $\gamma_{i}$ in the analogous way from $\alpha_{i}$ and $\beta_{i}$ instead of $a_{i}$ and $b_{i}$, we also see that $r\left(\gamma_{0}\right)=r\left(\gamma_{1}\right)=r\left(\gamma_{2}\right), \theta\left(\gamma_{1}\right)=-\theta\left(\gamma_{0}\right)$, and $\theta\left(\gamma_{2}\right)=2 \theta_{0}-\theta\left(\gamma_{0}\right)$. From $\left({ }^{* * *}\right)$ it follows that $\gamma_{i}\left(c_{i}\right)=1=\left\|\gamma_{i}\right\|=\left\|c_{i}\right\|$.

Now suppose that $r_{0}>1$; then $1 / r_{0}<1$ so $\theta\left(c_{0}\right)=\theta\left(a_{0}+b_{0}\right)>\theta_{0} / 2>\theta\left(\alpha_{0}+\beta_{0}\right)$ $=\theta\left(\gamma_{0}\right)$. (From the similar triangles $O a_{0} b_{0}$ and $O b_{\beta_{0}} a_{0}$ it is also clear that $\theta\left(c_{0}\right)+\theta\left(\gamma_{0}\right)=\theta_{0}$.) To get a contradictory relation let $c=\left(c_{1}+c_{2}\right) /\left\|c_{1}+c_{2}\right\|$ and $\gamma=\left(\gamma_{1}+\gamma_{2}\right) /\left\|\gamma_{1}+\gamma_{2}\right\|$. Then $\theta(c)=\theta\left(c_{1}+c_{2}\right)=\left[\theta\left(c_{1}\right)+\theta\left(c_{2}\right)\right] / 2$ since $r\left(c_{1}\right)=r\left(c_{2}\right)$. However $\left[\theta\left(c_{1}\right)+\theta\left(c_{2}\right)\right] / 2=\left[-\theta\left(c_{0}\right)+2 \theta_{0}-\theta\left(c_{0}\right)\right] / 2=\theta_{0}-\theta\left(c_{0}\right)\left(=\theta\left(\gamma_{0}\right)\right)$. Since $\theta\left(c_{0}\right)>\theta_{0} / 2>\theta\left(\gamma_{0}\right)$, it follows that

$$
\begin{aligned}
\theta(c) & =\theta_{0}-\theta\left(c_{0}\right)<\theta_{0} / 2<\theta_{0}-\theta\left(\gamma_{0}\right)=\left[\theta\left(\gamma_{1}\right)+\theta\left(\gamma_{2}\right)\right] / 2 \\
& =\theta\left(\gamma_{1}+\gamma_{2}\right)=\theta(\gamma) .
\end{aligned}
$$

Since $\theta(c)<\theta\left(c_{0}\right)$ it follows that

$$
\theta(\gamma) \leqq \theta\left(\gamma_{0}\right)<\theta_{0} / 2<\theta(\gamma)
$$

since this is impossible, $r_{0}$ is not greater than one. A similar argument shows that $r_{0}$ is not less than one so $r=1$ and $\left(1, \theta_{0}\right)$ is on $C$.

Applying this lemma by induction completes the proof of Theorem 3.2. The first step is to choose $\theta_{0}=\pi / 4$; this proves that $(1, \pi / 4)$ is on $C$ and that $C$ is symmetric about all the lines $\theta=m \pi / 4$. Applying the lemma repeatedly with $\theta_{0}=\pi / 2^{n}$ proves symmetry about the lines $\theta=m \pi / 2^{n}$ and that the points $\left(1, m \pi / 2^{n}\right)$ are on $C$ as well as on $E$. Since $C$ and $E$ coincide on a dense set, $C$ is $E$.

This proves Theorem 3.2; to complete the proof of Theorem 3.1 for reallinear spaces requires only the von Neumann-Jordan condition.

4. Characterizations involving uniform convexity and its dual, uniform 
flattening. A normed linear space $B$ is called uniformly convex if for each $\epsilon, 0<\epsilon \leqq 2$, there is a $\delta(\epsilon)>0$ such that $\left\|b_{1}+b_{2}\right\| \leqq 2(1-\delta(\epsilon))$ if $\left\|b_{1}-b_{2}\right\| \geqq \epsilon$ and $\left\|b_{1}\right\|=\left\|b_{2}\right\|=1$; the function $\delta$ is called the modulus of convexity of $B$. It is easily verified that any inner-product space is uniformly convex. If $\delta_{2}(\epsilon)=1-\left(1-(\epsilon / 2)^{2}\right)^{1 / 2}$, Clarkson [4] has shown that $\delta_{2}$ is the largest possible modulus of convexity in each inner-product space; in this section it will be proved that this modulus is as large as a modulus of convexity can be.

THEOREM 4.1. $B$ is uniformly convex with a modulus of convexity satisfying the inequality $\delta(\epsilon) \geqq \delta_{2}(\epsilon)$ for $0<\epsilon \leqq 2$ if and only if $B$ is an inner-product space and $\delta$ is identically equal to $\delta_{2}$.

The definition of $\delta_{2}$ gives the proof when $B$ is an inner-product space. On the other hand, if $\delta$ satisfies the inequality, take any two-dimensional subspace, let $C$ be the set of points of norm one in that subspace, and proceed, as in the first proof of $\S 2$, to let $E$ be the smallest ellipse around $C$, and to let $|b|$ be the norm in which $E$ is the unit sphere. If $x$ and $y \neq \pm x$ are points of contact of $C$ and $E$, let $\epsilon=|x-y|$ and $\epsilon^{\prime}=\|x-y\|$. Then $\epsilon^{\prime} \geqq \epsilon$ and

$$
\begin{aligned}
2\left(1-\delta_{2}(\epsilon)\right)=|x+y| & \leqq\|x+y\| \leqq 2\left(1-\delta\left(\epsilon^{\prime}\right)\right) \leqq 2\left(1-\delta_{2}\left(\epsilon^{\prime}\right)\right) \\
& \leqq 2\left(1-\delta_{2}(\epsilon)\right) .
\end{aligned}
$$

Since the ends are equal $|x+y|=\|x+y\|$ (and also $\epsilon^{\prime}=\epsilon$ ). As in the argument of $\S 2$, it follows that $C$ is $E$. The von Neumann-Jordan condition completes the proof of the main theorem.

As in [6] define $\eta(\epsilon)=\sup \left(2-\left\|b_{1}+b_{2}\right\|\right) /\left\|b_{1}-b_{2}\right\|$ where the supremum is taken for $\left\|b_{1}\right\|=\left\|b_{2}\right\|=1$ and $\left\|b_{1}-b_{2}\right\| \leqq \epsilon$. The space $B$ is called uniformly flattened if $\lim _{\epsilon \rightarrow 0} \eta(\epsilon)=0$. The function $\eta$ is called the modulus of flattening in $B$. In an inner product space $E$ the modulus of flattening $\eta_{2}$ is defined by $\eta_{2}(\epsilon)=2 \delta_{2}(\epsilon) / \epsilon$.

THEOREM 4.2. $B$ is an inner-product space if and only if $B$ is uniformly flattened with a modulus of flattening $\eta$ which satisfies the condition $\eta(\epsilon) \leqq \eta_{2}(\epsilon)$ if $0<\epsilon \leqq 2$.

The proof is similar to that 4.1 ; we need only use the unique ellipse of largest area inside $C$ as we used the ellipse of smallest area in the preceeding proof.

5. Characterizations by properties of orthogonality. In a recent note James [9] discussed certain definitions of orthogonality of points in a normed space and showed that if too many extra properties are allowed to any one of these definitions, then the space is forced to be an inner-product space.

In any inner-product space $B$, orthogonality of $b$ and $b^{\prime}$, that is, $\left(b, b^{\prime}\right)=0$, is characterized by any one of the following properties; they are listed with the labels used by James. 


$$
\begin{aligned}
\left\|b+b^{\prime}\right\| & =\left\|b-b^{\prime}\right\| \\
\left\|b+t b^{\prime}\right\| & =\left\|b-t b^{\prime}\right\| \text { for every real } t \\
\left\|b+b^{\prime}\right\|^{2} & =\|b\|^{2}+\left\|b^{\prime}\right\|^{2}
\end{aligned}
$$

(Isosceles orthogonality).

(Roberts' orthogonality).

(Pythagorean orthogonality).

James showed by a simple, two-dimensional example that in a general normed space these relations are no longer equivalent. He showed that (A) if orthogonality in either sense (1) or (3) is additive or homogeneous on one variable, then an inner product exists; and (B) if every two-dimensional subspace containing a point $b$ contains a point $b^{\prime}$ orthogonal in sense (2) to $b$, then an inner product exists.

In this section we apply the theorems of $\$ \S 2$ and 4 to round out James' study by showing that if either condition (1) or (3) implies the other, then there is an inner product.

THEOREM 5.1. If isosceles orthogonality implies Pythagorean orthogonality, that is, if $\left\|b+b^{\prime}\right\|=\left\|b-b^{\prime}\right\|$ implies $\left\|b+b^{\prime}\right\|^{2}=\|b\|^{2}+\left\|b^{\prime}\right\|^{2}$, then $B$ is an innerproduct space.

If $\|b\|=\left\|b^{\prime}\right\|=1$, then $b+b^{\prime}$ and $b-b^{\prime}$ are isosceles orthogonal. Hence these vectors are Pythagorean orthogonal and

$$
4=\|2 b\|^{2}=\left\|\left(b+b^{\prime}\right)+\left(b-b^{\prime}\right)\right\|^{2}=\left\|b+b^{\prime}\right\|^{2}+\left\|b-b^{\prime}\right\|^{2} .
$$

This is precisely condition $\left(^{*}\right)$ so, by Theorem $2.1, B$ is an inner-product space.

TheOREM 5.2. If Pythagorean orthogonality implies isosceles orthogonality in $B$, then $B$ is an inner-product space.

Given that $\left\|b+b^{\prime}\right\|^{2}=\|b\|^{2}+\left\|b^{\prime}\right\|^{2}$ we see that $\left\|b+b^{\prime}\right\|=\left\|b-b^{\prime}\right\|$ if and only if $\left\|b-b^{\prime}\right\|^{2}=\|b\|+\left\|b^{\prime}\right\|^{2}$. Hence Pythagorean orthogonality implies isosceles orthogonality if and only if $(C) b^{\prime}$ is Pythagorean orthogonal to $-b$ whenever it is Pythagorean orthogonal to $b$. Hence 5.2 is equivalent to the following lemma.

\section{LEMMA 5.3. If (C) holds, then $B$ is an inner-product space.}

The proof of this depends on Theorem 4.1. To compute the modulus of convexity in $B$ let a chord of the unit sphere of length $\epsilon<2$ be given and let $P$ be the plane containing the origin and the chord; let $y$ be the vector from the midpoint to one end of the chord. It is easily seen that for an arbitrary positive $k$ there is at least one $x$ in $P$ of norm $k$ which is Pythagorean orthogonal to $y$, for, as $x$ moves continuously around the curve of points of $P$ of norm $k$, the function $f(x)=\|x+y\|^{2}-k^{2}-\|y\|^{2}$ takes on both positive and negative values. Choose $k=\left(1-(\epsilon / 2)^{2}\right)^{1 / 2}$; then $\|x+y\|^{2}=\|x\|^{2}+\|y\|^{2}=1$ $=\|x-y\|^{2}$, so $x+y$ and $x-y$ are on the unit sphere in $P$. Now the given chord, the chord from $x+y$ to $x-y$, and the chord from $-x+y$ to $-x-y$ are all 
parallel and of length $\epsilon<2$. Hence the given chord must be one of the last two named; by proper choice of the sign of $x$ we have that the given chord has $x$ as its midpoint. The depth of $x$ below the surface of the unit sphere is $1-\|x\|=1-\left(1-(\epsilon / 2)^{2}\right)^{1 / 2}=\delta_{2}(\epsilon)$ for every $\epsilon<2$. Since $\delta_{2}(2)=1$ and $\delta$ is monotone, $\delta(\epsilon) \geqq \delta_{2}(\epsilon)$ for every $\epsilon \leqq 2$. By $4.1, B$ is an inner-product space.

Lemma 5.3 also offers a short proof of James' result that homogeneity of Pythagorean orthogonality implies the existence of an inner product, for this homogeneity implies (C). It may also be noted that James' condition $(\mathrm{H})$ of homogeneity of isosceles orthogonality is immediately equivalent to a condition given earlier by Ficken [7]. $B$ is an inner-product space if and only if (F) whenever $\|x\|=\|y\|$ and $p$ and $q$ are real, then $\|p x+q y\|=\|q x+p y\|$. (H) means that if $\left\|b+b^{\prime}\right\|=\left\|b-b^{\prime}\right\|$ and $t$ is real, then $\left\|t b+b^{\prime}\right\|=\left\|t b-b^{\prime}\right\|$. If $\|x\|=\|y\|$, then $b=x+y$ and $b^{\prime}=x-y$ are isosceles orthogonal; using $(\mathrm{H})$ and multiplying by a real $a$ gives $\left\|a t b+a b^{\prime}\right\|=\left\|a t b-a b^{\prime}\right\|$ or $\|a(t+1) x+a(t-1) y\|$ $=\|a(t-1) x+a(t+1) y\|$. For any $p \neq q$ the equations $a(t+1)=p$ and $a(t-1)=q$ have the solutions $a=(p-q) / 2$ and $t=(p+q) /(p-q)$; (F) follows immediately. Similarly, if $(\mathrm{F})$ holds and $b$ is isosceles orthogonal to $b^{\prime}$, the substitutions $b+b^{\prime}=x, b-b^{\prime}=y, 2 p-1=t$, and $q=p-1$ in $(\mathrm{F})$ give $(\mathrm{H})$.

A geometric theorem weaker than James' results is:

THEOREM 5.4. $B$ is an inner-product space if and only if the set of points equidistant from any pair of given points is flat.

This property is obvious in an inner-product space. If it holds in $B$ then for each $B$ the set of points equidistant from $b$ and $-b$ is linear, hence homogeneous. By James' result (A) quoted before (5.1), $B$ is an inner-product space.

6. Characterization by symmetry of normality. Say that $x$ normal to $y$ means that $\|a x+y\| \geqq\|y\|$ for every real $a$. In an inner-product space $x$ is normal to $y$ if and only if $(x y)=0$; hence normality is a symmetric relationship in an inner-product space. In two-dimensional spaces the converse is not true; for a three-or-more-dimensional space, however, symmetry of normality is characteristic of inner-product spaces. This section gives, first, a method of constructing all two-dimensional spaces in which normality is symmetric, and, second, proves that in a space of dimension greater than two symmetry of normality makes the space an inner-product space.

The method of constructing a two-dimensional norm with symmetry of normality is, essentially, to take a fairly general norm in first and third quadrants and to fit in the appropriate part of the conjugate norm in the second and fourth quadrants. More precisely, let $B$ be any two-dimensional normed space and let $C$ be the set of points of norm one. Theorem 1 of the preceding paper [5] shows that there exist at least one pair of points $k_{1}, b_{2}$, on $C$ such that $b_{1}$ is normal to $b_{2}$ and $b_{2}$ is normal to $b_{1}$. Set up an $x, y$ co- 
ordinate system in $B$ with $b_{1}$ and $b_{2}$ at $(1,0)$ and $(0,1)$, respectively, and define $f(x, y)=\|b\|$ if $b=(x, y)$.

Represent the conjugate space $B^{*}$ of $B$ in the usual manner as the set of all pairs $(p, q)$ such that $\beta$ corresponds to $(p, q)$ if $\beta(b)=p x+q y$ for every $b$ in $B$. Then for $\beta=(p, q)$ define $\phi(p, q)=\|\beta\|=\sup _{(x, y) \neq(0,0)}|p x+q y| / f(x, y)$. The condition that the line $\left\{b \mid \beta(b)=\left\|b_{0}\right\| \cdot\|\beta\|\right\}$ be a line of support to the sphere $\left\{b \mid\|b\|=\left\|b_{0}\right\|\right\}$ is that $\left|\beta\left(b_{0}\right)\right|=\|\beta\| \cdot\left\|b_{0}\right\|$. This translates into the equation

$$
\left|p x_{0}+q y_{0}\right|=\phi(p, q) f\left(x_{0}, y_{0}\right) .
$$

Now in order that $\left(x_{1}, y_{1}\right)$ be normal to $\left(x_{0}, y_{0}\right)$ it is necessary that it lie in a line through the origin parallel to a line of support at $\left(x_{0}, y_{0}\right)$ to $\{b \mid\|b\|$ $\left.=f\left(x_{0}, y_{0}\right)\right\}$; that is, that there exist $p$ and $q$ satisfying (1) for which $p x_{1}+q y_{1}$ $=0$. Hence $(p, q)$ is a multiple of $\left(y_{1},-x_{1}\right)$ and condition (1) shows that $\left(x_{1}, y_{1}\right)$ is normal to $\left(x_{0}, y_{0}\right)$ if and only if

$$
\left|y_{1} x_{0}-x_{1} y_{0}\right|=\phi\left(y_{1}, x_{1}\right) f\left(x_{0}, y_{0}\right) .
$$

Now define a new norm $|b|$ by the equations $|(x, y)|=f(x, y)$ if $x y \geqq 0$ (that is, if $(x, y)$ is in the first or third quadrant), $|(x, y)|=\phi(y,-x)$ if $x y \leqq 0$.

From the choice of axes on the original curve $C$ it is clear that on this new curve $\left(x_{1}, y_{1}\right)$ is normal to $\left(x_{0}, y_{0}\right)$ only in case $x_{1} y_{1}$ and $x_{0} y_{0}$ have opposite signs. Hence when $x_{0} y_{0} \geqq 0$ equation (2) says that relative to this new norm a point $\left(x_{1}, y_{1}\right)$ is normal to $\left(x_{0}, y_{0}\right)$ if and only if

$$
\left|y_{1} x_{0}-x_{1} y_{0}\right|=\left|\left(x_{1}, y_{1}\right)\right| \cdot\left|\left(x_{0}, y_{0}\right)\right| \text {. }
$$

We wish now to establish the same condition for normality to points in the second and fourth quadrants. If $x_{1} y_{1} \leqq 0$, then $\left|\left(x_{1}, y_{1}\right)\right|=\phi\left(y_{1},-x_{1}\right)$. If an element of the new conjugate space is located as before by $(p, q)$ and defines a line of support at any point in the second or fourth quadrants, then the new norm is given by

$$
\begin{aligned}
F(p, q) & =\sup _{(x, y) \neq(0,0)}|p x+q y| /|(x, y)|=\sup |p x+q y| / \phi(y,-x) \\
& =\sup |q y+(-p)(-x)| / \phi(y,-x) .
\end{aligned}
$$

Thinking for the moment of $(y,-x)$ as lying in $B^{*}$ and $(q,-p)$ as in $B^{* *}$, this is precisely the formula for the norm in $B^{* *}$ of the point $(q,-p)$. Since any two-dimensional space is reflexive, it follows that

$$
F(p, q)=f(q,-p) \text {. }
$$

Now for $(p, q)$ to define a line of support at $\left(x_{1}, y_{1}\right)$ we have the usual condition

$$
\left|p x_{1}+q y_{1}\right|=F(p, q)\left|\left(x_{1}, y_{1}\right)\right|=f(q,-p) \phi\left(y_{1},-x_{1}\right) .
$$

As before for a point $\left(x_{2}, y_{2}\right)$ to be normal to $\left(x_{1}, y_{1}\right)$ we have $p x_{2}+q y_{2}=0$ or 
$(p, q)$ a multiple of $\left(y_{2},-x_{2}\right)$. Then for normality in this quadrant we get

$$
\left|y_{2} x_{1}-x_{2} y_{1}\right|=f\left(-x_{2},-y_{2}\right) \phi\left(y_{1},-x_{1}\right)=f\left(x_{2}, y_{2}\right) \phi\left(y_{1},-x_{1}\right)
$$

and the condition for normality holds independent of the quadrant concerned. Hence for $\left(x_{1}, y_{1}\right)$ normal to $\left(x_{0}, y_{0}\right)$ relative to the new norm we have

$$
\left|y_{1} x_{0}-x_{1} y_{0}\right|=\left|\left(x_{1}, y_{1}\right)\right| \cdot\left|\left(x_{0}, y_{0}\right)\right|
$$

so

$$
\left|y_{0} x_{1}-x_{0} y_{1}\right|=\left|\left(x_{0}, y_{0}\right)\right| \cdot\left|\left(x_{1}, y_{1}\right)\right| .
$$

Hence normality is reciprocal for $|\cdots|$.

If $C$ is the unit sphere in a two-dimensional space, call the short arc around $C$ from $b_{1}$ to $b_{2}$ a quadrant of $C$ if each $b_{i}$ is normal to the other. Theorem 1 of the preceding paper [5] can be rephrased in terms of this definition as: If $C$ is the unit sphere in any two-dimensional real-linear normed space, there is at least one way to divide $C$ into quadrants. Representing both $C$ and the unit sphere, $C^{*}$, of the conjugate space in an $x, y$ coordinate system as above, where $(1,0)$ and $(0,1)$ are the ends of a quadrant of $C$, the construction above asserts that any quadrant of the unit sphere $C$ in a two-dimensional normed space is a quadrant of a curve $C_{1}$ which is the unit sphere of a two-dimensional space in which normality is symmetric; the first and third quadrants of $C_{1}$ are those of $C$, the second and fourth quadrants of $C_{1}$ are the first and third of $C^{*}$ (merely rotated by $\pi / 2$ if the $x, y$ coordinate system is first made rectangular by introducing an appropriate inner product). The examples of James [10] are special cases of this construction using for $C$ any curve

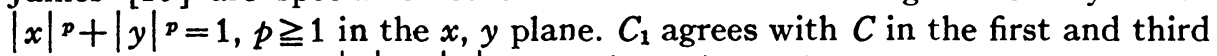
quadrants and with $|x|^{q}+|y|^{q}=1,1 / p+1 / q=1$, in the second and fourth. In the limiting case, $p=1, C_{1}$ is affine to the regular hexagon which is, therefore, the unit sphere in a space with symmetry of normality but not strict convexity.

It may be of interest to note that this special case $p=1$ was the starting point for my original proof that any quadrant of $C$ is a quadrant of a $C_{1}$ with symmetry of normality. The main step in that proof is to show that if a given symmetric, convex polygon $P$ has symmetry of normality and if the number of vertices of $P$ is increased by replacing two parallel sides of $P$ by the other sides of triangles so added on that the new polygon is also convex and symmetric, then by appropriately trimming two opposite corners in each of the remaining quadrants of $P$, symmetry of normality can be regained. Now start wich a quadrant of $C$ and a coordinate system in which $(1,0)$ and $(0,1)$ are ends of the quadrant; in the example, $p=1$, above, we have a polygon with symmetry of normality and with one quadrant inscribed in the given quadrant of $C$. Adding vertices one at a time from a set of points dense in the given quadrant gives a sequence of polygons with symmetry of normality 
and with first quadrants converging to the given quadrant of $C$. This sequence converges to the desired $C_{1}$.

To show that every two-dimensional norm in which normality is reciprocal can be constructed in this way we need a uniqueness theorem which will also have applications in the later proofs in three-dimensional spaces.

Lemma 6.1. Let $\|b\|$ and $|b|$ be norms in a two-dimensional space, let $C$ and $C^{\prime}$ be the corresponding sets of points of norm one, and suppose that for each norm normality is symmetric; if on $C$ there exist two points $b_{1}$ and $b_{2}$ normal to $b_{1}$ such that $C$ and $C^{\prime}$ agree along the arc from $b_{1}$ to $b_{2}$, then $C$ and $C^{\prime}$ are identical; that is, $\|b\|=|b|$ for every $b$.

By an affine transformation we can assume that the curves $C$ and $C^{\prime}$ are defined by equations in polar coordinates $r=f(\theta)$ and $r=\phi(\theta)$ such that $f(\theta)=\phi(\theta)$ for $0 \leqq \theta \leqq \pi / 2$. At any value of $\theta$ in the second quadrant the symmetry of normality asserts that the half tangents to $C$ are parallel to those to $C^{\prime}$. Hence $d(\log f(\theta)-\log \phi(\theta)) / d \theta=0$ so $f(\theta) / \phi(\theta)=$ constant for $\pi / 2 \leqq \theta \leqq \pi$. As $f(\pi / 2)=\phi(\pi / 2), f(\theta)=\phi(\theta)$ for all $\theta$.

More briefly we have proved if $C_{1}$ and $C_{2}$ both have symmetry of normality and agree along a quadrant of one of them, then $C_{1}$ and $C_{2}$ are identical.

COROLlaRy 6.2. If in the two-dimensional normed space $B$ normality is symmetric and $b$ is normal to $b^{\prime}$, then the whole unit sphere is determined by its shape in the quadrant from $b$ to $b^{\prime}$.

COROLlaRY 6.3. If in a two-dimensional normed space $B$ normality is symmetric, then the processes described before Lemma 6.1 leave the norm invariant. Hence all two-dimensional norms in which normality is symmetric can be constructed by the process.

We now have enough information on two-dimensional norms to prove the following theorem.

THEOREM 6.4. If the real-linear space $B$ has more than two linearly independent elements and if normality is symmetric, then $B$ is an inner-product space.

It suffices to prove the theorem if $B$ is precisely three-dimensional. For the simplest proof of Theorem 6.4, it suffices to use a theorem of Kakutani [12] that if there is a projection of norm no greater than one on every closed linear subspace of $B$, then $B$ is an inner product space $\left({ }^{4}\right)$. As before, we need consider only the three-dimensional case. If $P$ is any plane through the origin, let $x$ be a point of contact of the unit sphere with the plane of support parallel to $P$. Then every point in $P$ is normal to $x$; by symmetry of normality $x$ is normal to every point of $P$. Therefore the projection on $P$ along $O x$ is of norm one. Obviously projections of norm no greater than one exist on zero-,

( $)$ An elementary proof of this theorem was given by Phillips [13]. 
one-, and three-dimensional subspaces; Kakutani's theorem now asserts the existence of an inner product.

An alternative proof reduces 6.4 to a theorem of G. Birkhoff [2] which differs from 6.4 only in having the additional hypothesis of strict convexity. Again we need only prove the three-dimensional case. Clearly in the presence of symmetry of normality strict convexity is equivalent to the existence of a unique tangent plane at every point of the unit sphere $C$ of $B$. If there were a point $x$ on $C$ with more than one plane of support touching $C$ at $x$, then the supporting cylinder $\Sigma$ of $C$ parallel to $O x$ would meet $C$ in a band around $C$ included between the two half-cones at $O$ parallel to the half-cones of half tangents at $x$ and $-x$. It would then be simple to find a plane $\Pi$ through $O$ such that $\Pi \cap C$ and $\Pi \cap \Sigma$ agreed along more than a quadrant of $\Pi \cap C$ but were not identical. Since $\Pi \cap \Sigma$ is affine to any cross section of $C$ lying in $\Sigma \cap C$, this would contradict 6.1 . Hence symmetry of normality and three-dimensionality imply strict convexity. G. Birkhoff's result [2] now asserts that $B$ is an inner-product space.

In my referee's report on the manuscript [10] I mentioned these two proofs of 6.4 to James; he had independently proved this same theorem [10, Theorem 1] by an algebraic, rather than geometric, reduction to G. Birkhoff's result.

7. Complex-linear spaces. There is no direct application of the proofs used in the real-linear spaces to complex-linear normed spaces but we can use the theorems we have proved in the real-linear case to derive the corresponding theorems in the complex-linear case. These proofs depend on certain elementary relations between each complex-linear space and an associated real-linear space. Only enough discussion is given here for the proofs of these results; I hope to give other applications in a later paper $\left(^{(5)}\right.$.

If $B$ is a complex-linear space, there is associated to $B$ a unique reallinear space $A$ whose elements are the elements of $B$ and whose addition is also that in $B$; if $x$ is a real number and $b \in B$, the multiple $x b$ in $A$ is just the complex multiple $(x+i 0) b$ in $B$. Roughly speaking $A$ is what is left of $B$ if the multiplication by non-real complex numbers is just forgotten. If $\|b\|$ is a norm in $B$, then the same norm is to be used in $A$.

As is usual for complex numbers if $z=x+i y$, we let $R z=x, I z=y$, and $\bar{z}=x-i y$. If $\beta \in B^{*}$, that is, if $\beta$ is a complex-valued, complex-linear function on $B$, define $R \beta$, the real part of $\beta$, by $\alpha=R \beta$ if $\alpha(b)=R(\beta(b))$ for every $b$. Obviously $\alpha$ is a real-valued, real-linear function defined on $A$, so $R \beta \in A^{*}$, the set of real-linear, real-valued functions on $A$, if $\beta \in B^{*}$. More than this is true.

THEOREM 7.1. The function $R$ defined by $R \beta=\alpha$ if $\alpha(b)=R(\beta(b))$ is a one-to-

(5) Although 7.1 and 7.2 have not been stated formally before, the facts embodied in them appear in various places. See, for example, [3], [8], and [11]. 
one, real-linear, norm-preserving function between all of $A^{*}$ and all of $B^{*}$.

The algebraic part of this follows easily from the observation that $\beta(b)=\alpha(b)-i \alpha(i b)$ is the only suitable relation for returning from $\alpha$ to $\beta$.

To prove $\|\alpha\|=\|\beta\|$ if $\alpha=R \beta$, we see first that $|\alpha(b)|=|R(\beta(b))| \leqq|\beta(b)|$ for every $b$ so $\|\alpha\| \leqq\|\beta\|$. On the other hand if $\beta(b)=r e^{i \theta}$, then $\beta\left(b e^{-i \theta}\right)=e^{-i \theta} r e^{i \theta}$ $=r=|\beta(b)|$. Since $\beta\left(b e^{-i \theta}\right)$ is real $\left|\alpha\left(b e^{-i \theta}\right)\right|=\left|\beta\left(b e^{-i \theta}\right)\right|=|\beta(b)|$ while $\left\|b e^{-i \theta}\right\|$ $=\|b\|$ so $\|\alpha\|=\sup \|b ! \leq 1|\alpha(b)|=\sup |b| \leq 1|\beta(b)|=\| \beta \|$.

Before we can prove our next result we recall the definition of a complex inner product. A complex-valued function $\left[b, b^{\prime}\right]$ defined for pairs of points in a complex-linear space is called a complex inner product if it satisfies the three conditions

C1. $[b, b] \geqq 0$, and equality holds if and only if $b=0$.

C2. $\left[b, b^{\prime}\right]=\left[\overline{b^{\prime}, b}\right]$ for every $b$ and $b^{\prime}$ in $B$.

C3. $\left[z_{1} b_{1}+z_{2} b_{2}, b_{3}\right]=\left[z_{1} b_{1}, b_{3}\right]+\left[z_{2} b_{2}, b_{3}\right]$ for every choice of $b_{i}$ in $B$ and complex numbers $z_{i}$.

A complex-linear normed space $B$ is a complex inner-product space if there exists a complex inner product satisfying $\|b\|^{2}=[b, b]$. The next theorem says that a complex-linear space has a complex inner-product if and only if it has a real inner product.

TheOREM 7.2. A normed complex-linear space $B$ is a complex inner-product space if and only if the associated real-linear space $A$ is a real inner-product space. When this happens the inner products $\left(b, b^{\prime}\right)$ in $A$ and $\left[b, b^{\prime}\right]$ in $B$ are related by the equations $\left(b, b^{\prime}\right)=R\left(\left[b, b^{\prime}\right]\right)$ and $\left[b, b^{\prime}\right]=\left(b, b^{\prime}\right)-i\left(i b, b^{\prime}\right)$.

If $B$ is a complex inner-product space, it is clear that $\left(b, b^{\prime}\right)=R\left(\left[b, b^{\prime}\right]\right)$ defines a real inner product in $A$; since $\|b\|^{2}=[b, b]$ is real, $\|b\|^{2}=(b, b)$ and $A$ is a real inner-product space.

If $\left(b, b^{\prime}\right)$ is a real inner-product in $A$, methods used in [11] show that $\left(b, i b^{\prime}\right)=-\left(i b, b^{\prime}\right)$ for every $b$ and $b^{\prime}$. Direct computation with this and properties R1-R3 give $\mathrm{C} 1-\mathrm{C} 3$.

Theorems 7.1 and 7.2 give all the necessary machinery for the proofs of the complex cases of the preceding theorems.

Theorem 7.3. A complex-linear normed space $B$ is an inner-product space if and only if one (or all) of the following conditions holds:

(1) Condition $\left({ }^{*}\right)$.

(2) Condition $\left({ }^{* *}\right)$ or $\left({ }^{* * *}\right)$.

(3) $\delta(\epsilon) \geqq \delta_{2}(\epsilon)$ whenever $0<\epsilon \leqq 2$, or $\eta(\epsilon) \leqq \eta_{2}(\epsilon)$ whenever $0<\epsilon \leqq 2$.

(4) $\left\|b+b^{\prime}\right\|=\left\|b-b^{\prime}\right\|$ implies $\left\|b+b^{\prime}\right\|^{2}=\|b\|^{2}+\left\|b^{\prime}\right\|^{2}$.

(5) $\left\|b+b^{\prime}\right\|^{2}=\|b\|^{2}+\left\|b^{\prime}\right\|^{2}$ implies $\left\|b+b^{\prime}\right\|=\left\|b-b^{\prime}\right\|$.

(6) Normality is symmetric in $B$.

(7) Additivity or homogeneity of isosceles or Pythagorean orthogonality (James) or the condition (F) of Ficken. (Where scalar multiples occur the condi- 
tions may be phrased with either real or complex numbers.)

Conditions (3) through (7) and (1) depend only on the norm in $B$. Hence they hold in the associated real-linear $A$ if and only if they hold in $B$. By the preceding sections any one of these conditions but (6) is equivalent to the existence of a real inner product in $A$. By 7.2 each condition is equivalent to the existence of a complex-inner product in $B$.

For the condition (6) we note that if $B$ has two or more complex-linearly independent elements, then $A$ is at least four-dimensional and (6) makes $A$ a real-inner-product space. If $B$ has not as many as two independent elements, $B$ is either a trivial space with only the point 0 or else $B$ is equivalent to the complex plane. In either case (6) holds and $B$ is a complex inner-product space; hence for complex-linear spaces (6) is equivalent to requiring $B$ to be an inner-product space. Recall that $x$ normal to $y$ was defined to mean $\|a x+y\| \geqq\|y\|$ for every real $a$. If we define $x$-complex-normal to $y$ to mean that this relation holds for all complex $a$, it is not known whether symmetry of complex normality in $B$ implies existence of an inner product.

Conditions $\left({ }^{* *}\right)$ and $\left({ }^{* * *}\right)$ use points of the conjugate space $B^{*}$ so we will need 7.1 as well as 7.2. Theorems 7.2 and 3.1 assert that $B$ is a complex innerproduct space if and only if the condition $\left({ }^{* *}\right)$ holds in $A$; to complete the proof we need only show that $\left(^{* *}\right)$ holds in $A$ if and only if it holds in $B$; the direct equivalence of $\left({ }^{* *}\right)$ and $\left({ }^{* * *}\right)$ is proved in the same way in $B$ as in $A$.

That condition $\left({ }^{* *}\right)$ holds in $A$ means that if $\left\|\alpha_{j}\right\|=\left\|b_{j}\right\|=\alpha_{j}\left(b_{j}\right)=1$, $j=1,2$, then $\left\|\alpha_{1}+\alpha_{2}\right\| \cdot\left\|b_{1}+b_{2}\right\|+\left\|\alpha_{1}-\alpha_{2}\right\| \cdot\left\|b_{1}-b_{2}\right\|=4$. Now if $\left\|\beta_{j}\right\|=\left\|b_{j}\right\|$ $=\beta_{j}\left(b_{j}\right)=1$, set $\alpha_{j}=R \beta_{j}$; then $\alpha_{j}\left(b_{j}\right)=R\left(\beta_{j}\left(b_{j}\right)\right)=1$ so, since $R$ preserves norms, $\left({ }^{* *}\right)$ holds in $B$ if it holds in $A$. Now if $\left({ }^{* *}\right)$ holds in $B$ and $\left\|\alpha_{j}\right\|=\left\|b_{j}\right\|=\alpha_{j}\left(b_{j}\right)=1$, choose $\beta_{j}=R^{-1} \alpha_{j}$. Then $\left\|\beta_{j}\right\|=\left\|\alpha_{j}\right\|=1$ and $\beta_{j}\left(b_{j}\right)$ $=\alpha_{j}\left(b_{j}\right)-i \alpha_{j}\left(i b_{j}\right)$. Hence $1=\alpha_{j}\left(b_{j}\right)=\left\|\alpha_{j}\right\| \cdot\left\|b_{j}\right\|=\left\|\beta_{j}\right\| \cdot \| b_{j}|\geqq| \beta_{j}\left(b_{j}\right) \mid=\left(\alpha_{j}\left(b_{j}\right)^{2}\right.$ $\left.+\alpha_{j}\left(i b_{j}\right)^{2}\right)^{1 / 2}=\left(1+\alpha_{j}\left(i b_{j}\right)^{2}\right)^{1 / 2} \geqq 1$. Hence $\alpha_{j}\left(i b_{j}\right)=0$ and $\beta_{j}\left(b_{j}\right)=1$; now $\left.{ }^{(* *}\right)$ in $B$ obviously implies it in $A$.

\section{BIBLIOGRA PHY}

1. S. Banach, Theorie des operations lineaires, Monografje Matematyczne, Warsaw, 1932.

2. G. Birkhoff, Orthogonality in linear metric spaces, Duke Math. J. vol. 1 (1935) pp. 169172.

3. H. F. Bohnenblust and A. Sobczyk, Extensions of functionals on complex-linear spaces, Bull. Amer. Math. Soc. vol. 44 (1938) pp. 91-93.

4. J. A. Clarkson, Uniformly convex spaces, Trans. Amer. Math. Soc. vol. 40 (1936) pp. $396-414$.

5. M. M. Day, Polygons circumscribed about closed convex curves, Trans. Amer. Math. Soc. vol. 315-319.

6. - Uniform convexity in factor and conjugate spaces, Ann. of Math. (2) vol. 45 (1944) pp. 375-385.

7. F. A. Ficken, Note on the existence of scalar products in normed linear spaces, Ann. of Math. (2) vol. 45 (1944) pp. 362-366. 
8. M. Fréchet, Sur lá definition axiomatique d'une classe d'espaces vectoriels distanciés applicables vectoriellement sur l'espace de Hilbert, Ann. of Math. (2) vol. 36 (1935) pp. 705-718.

9. R. C. James, Orthogonality in normed linear spaces, Duke Math. J. vol. 12 (1945) pp. 291-302.

10. - Inner products in normed linear spaces, Bull. Amer. Math. Soc. vol. 53 (1947) pp. 559-566.

11. P. Jordan and J. von Neumann, On inner products in linear metric spaces, Ann. of Math. (2) vol. 36 (1935) pp. 719-723.

12. S. Kakutani, Some characterizations of Euclidean space, Jap. J. Math. vol. 16 (1934) pp. 93-97.

13. R. S. Phillips, A characterization of Euclidean spaces, Bull. Amer. Math. Soc. vol. 46 (1940) pp. 930-933.

\section{UNIVERSITY OF IILLINOIS,}

URBANA, ILL. 